\title{
MECHANICS OF AIRFLOW IN HEALTH AND IN EMPHYSEMA
}

\section{BY HOWARD DAYMAN}

\author{
(From the Tuberculosis Service, Edward J. Meyer Memorial Hospital, Buffalo, N. Y.)
}

(Submitted for publication March 5, 1951 ; accepted August 13, 1951)

Essential manifestations of pulmonary emphysema are retarded expiratory airflow and incomplete expiration (1-3). These occur even though the patient makes a strenuous muscular effort to expel the vital capacity air as quickly and completely as possible. Inspiration is, by contrast, relatively unimpeded. The circumstances suggest the presence of expiratory obstruction, which is aggravated by increasing the expulsive force, in other words, a check valve type of obstruction. To determine whether such a mechanism exists we employed the method of Neergaard and Wirz (4) which, under suitable conditions, provides a continuous record of pleural pressure and rate of airflow. From these one may compute pulmonary pressure, by which we mean the pressure in the terminal air spaces; together with lung tension. The latter designates the net elastic recoil of the lungs, or "elastance" (5), expressed in centimeters of water pressure, under static conditions, at a particular lung volume. It represents the pressure required to maintain the lungs at that degree of distension when no air is passing in or out of the organs.

Relevant observations on the lungs at necropsy, and on the gross behavior of the major airways during forceful expiration were also included.

\section{METHOD}

As modified by us, the method is as follows: By means of a rubber mouth piece the subject breathes through a tube $30 \mathrm{~cm}$. long and $2.5 \mathrm{~cm}$. in diameter (Pneumotachygraph Figure 1). Within the lumen of the breathing tube are three bundles of thin brass tubing to prevent turbulence. Opposite small gaps between the bundles are side tubes to which short lengths of rubber tubing are attached. A cylindrical light bulb keeps the tube above body temperature to prevent condensation of moisture. An outer jacket enclosed both tube and bulb.

The difference in pressure between the two points where the side tubes are attached is a measure of the rate of airflow. This pressure difference is recorded by a differential tambour. The upper half of the tambour is of conventional shape, the lower half is provided with an airtight enclosure containing two glass windows. To the rubber membrane is attached an inverted aluminum fork bearing on its prongs a fine quartz fibre at the level of the window. The shadow of the cross hair with the beam of light is directed through a lens system against a vertical slit behind which electrocardiograph paper is being drawn at constant rate. When set up for actual use the natural frequency of the tambour is 3.4 complete cycles/sec. The resistance to breathing afforded by the airflow tube is negligible with rates up to $500 \mathrm{cc} . / \mathrm{sec}$. At faster rates it contributes a minor but measurable resistance. The exact resistance in the breathing tube is determined by having a subject breathe in and out through the tube at various rates while the pressure in the mouthpiece is being simultaneously measured by an optically recorded tambour. For a particular rate of airflow such pressure represents the resistance in the breathing tube itself, and in an actual experiment such a pressure is subtracted from the intrapulmonary pressure to calculate the resistance in the respiratory tract of the patient.

The pneumotachygraph was calibrated by having a subject breathe through the tube while the distal end was connected to a basal metabolism spirometer, movement of the latter being recorded on a fast moving drum. Synchronized timers marked both tracings. The slope of the spirometer tracing represents the rate of airflow. Numerous breaths were tested, at various rates of airflow, both inspiration and expiration. An attempt was made to hold the rate of airflow constant for a substantial part of a particular breath since the metabolism spirometer was not as sensitive to change in rate as was the pneumotachygraph. From periods of constant airflow many calibration points were determined and the curve was smoothed by the use of moving averages.

The basal metabolism spirometer can be expected to record the total volume of a breath with reasonable accuracy. This provides a means of testing the accuracy of the optical method. On a photographic tracing of an inspiration or expiration, the volume inhaled may be computed for each $1 / 10$ second during the breath. The sum of such increments should equal the volume of the breath. Tested from time to time during the course of the experiments, the average difference between volumes simultanously recorded by the spirometer and by the optical method was $\pm 4 \%$ (range $3 \%$ to $8 \%$ ).

Pleural pressures were recorded by means of a simple tambour provided with a fork and cross hair, the shadow of the latter being cast on another strip of electrocardiograph paper wound on the same drum and marked with a synchronized timer. With a 14 gauge needle attached to the system the natural frequency of the tambour was 9 complete cycles/sec. The entire apparatus is conveniently managed by one operator. Patients were tested while reclining at an angle of $45^{\circ}$. The needle was in- 


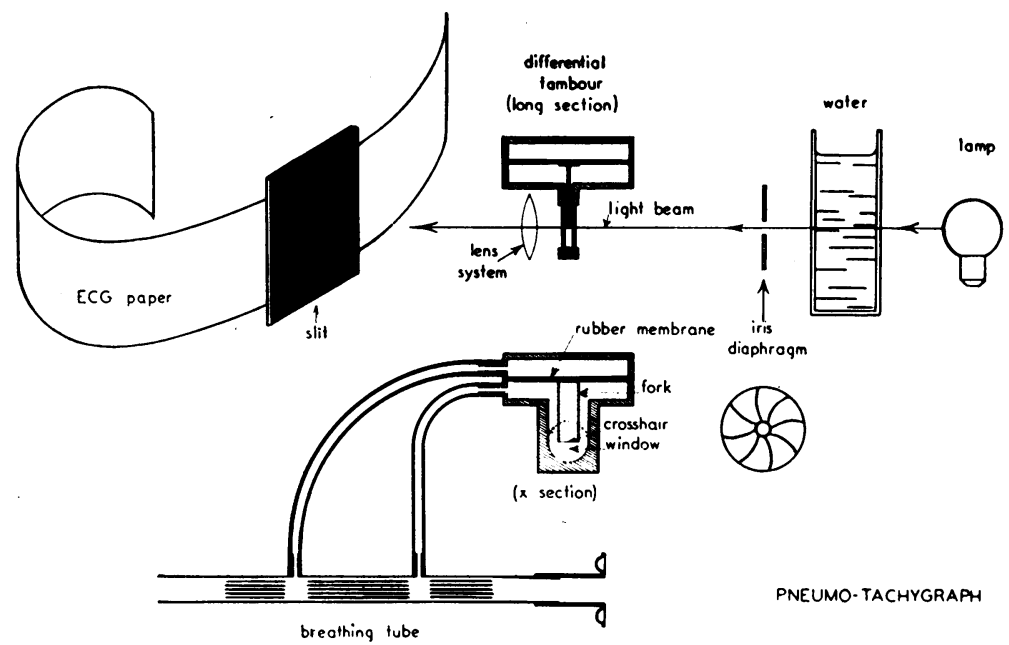

FIG. 1

serted in the second interspace except when otherwise indicated. Photographic tracings are illustrated in Figure 2.

Calculations: The deflections of the tracings from the zero line were measured at $1 / 10$ second intervals, converted to standard units of cc. per second or $\mathrm{cm}$. water pressure and transferred to graph paper. Lung tension can be measured directly at the instant of apnea between breaths. The determination of lung tension for intervening points depends on the frequently recorded observation (2-4) that lung tension, at least in normal functioning lungs, varies directly with lung volume. Change in lung volume for each breath was plotted on graph paper, as in Figure 3B, the straight line representing the change in volume un- corrected for variations in rate of airflow. The curved line represents the corrected volume change derived by plotting the increments at $1 / 10$ second intervals. The pleural pressure, Figure $3 \mathrm{C}$, is plotted to the same time scale. A straight line connecting the static pressures at the beginning and end of the breath will represent the uncorrected lung tension for intervening points. To obtain the corrected lung tension at each $1 / 10$ second interval one offsets each point the same amount of time as was offset in the corrected curve describing the change of lung volume. For example, $a-b$ equals $a^{\prime}-b^{\prime}$, and $c-d$ equals $c^{\prime}-d^{\prime}$.

Pulmonary pressure is determined by measuring the difference between corrected lung tension and pleural

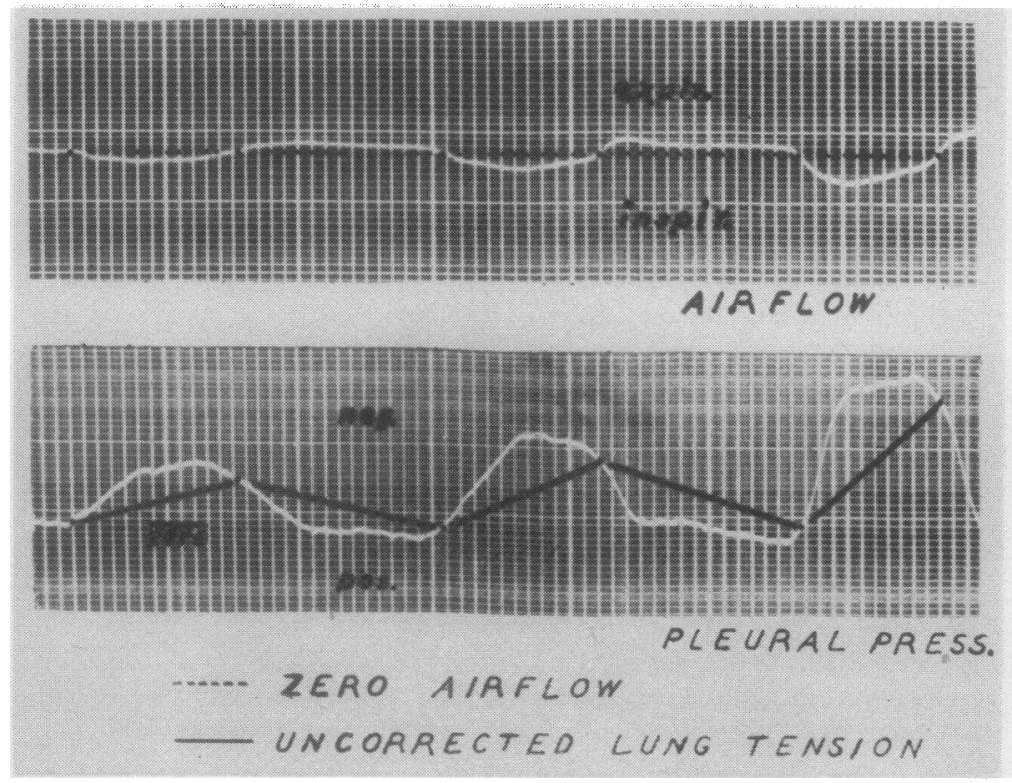

FIG. 2 
pressure at a given moment. During inspiration pleural pressure overcomes lung tension drawing air into the lungs. During expiration the reverse is true. See Figure 4.

Resistance in the respiratory tract at a particular moment during either inspiration or expiration is calculated by the formula:

Where:

$$
\mathrm{R}=\frac{(\mathrm{Pp}-\mathrm{pb}) \times 1,000}{\mathrm{~A}} .
$$

$\mathrm{R}=$ resistance, expressed in terms of $\mathrm{cm} . \mathrm{HOH}$ pressure necessary to cause $1,000 \mathrm{cc} . / \mathrm{sec}$. airflow.

$\mathrm{A}=$ airflow in cc./sec.

$\mathrm{Pp}=$ pulmonary pressure, in $\mathrm{cm} . \mathrm{HOH}$.

$\mathrm{pb}=$ fall of pressure across breathing tube at that particular rate of airflow, in $\mathrm{cm}$. $\mathrm{HOH}$.

The coefficient of lung tension is determined by comparing the change in lung tension with the change in lung volume for a series of breaths of varying size and is expressed

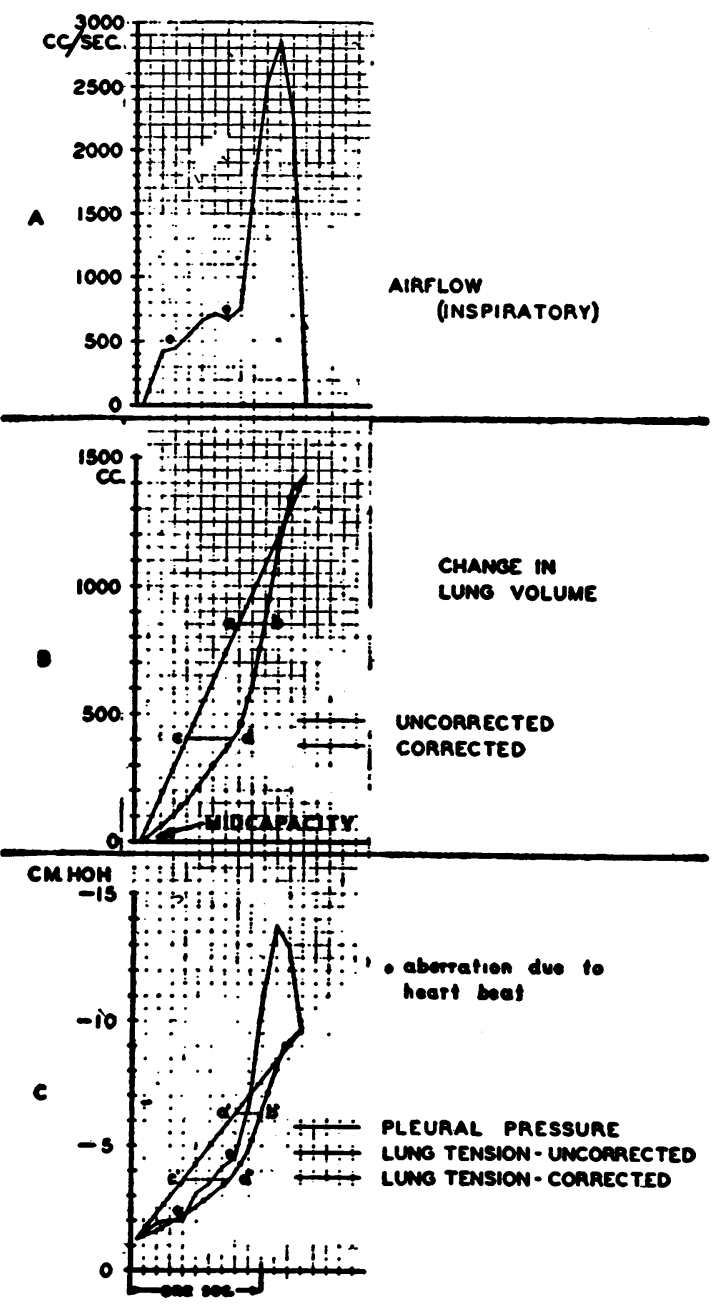

Fig. 3
TABLE I

\begin{tabular}{|c|c|c|c|}
\hline Case No. & $\begin{array}{c}\text { Number of } \\
\text { breaths } \\
\text { studied }\end{array}$ & $\begin{array}{l}\text { Coefficient } \\
\text { of lung } \\
\text { tension* }\end{array}$ & Comment \\
\hline 1 & 10 & .78 & Normal \\
\hline 2 & 6 & .86 & Normal \\
\hline 3 & 6 & .79 & Normal \\
\hline 3 & 6 & .77 & $\begin{array}{l}\text { After } 500 \text { cc. pneumo. } \\
\text { refill }\end{array}$ \\
\hline 4 & 9 & .78 & Normal \\
\hline 4 & 7 & .60 & $\begin{array}{l}\text { After } 500 \text { cc. pneumo. } \\
\text { refill }\end{array}$ \\
\hline 5 & 12 & .69 & Normal \\
\hline 6 & 8 & .52 & Chronic bronchitis \\
\hline 7 & 4 & 1.41 & Isolated bullae \\
\hline 8 & 8 & A. $R$. & $\begin{array}{l}\text { Advanced emphysema. } \\
\text { Maximum lung tension } \\
\text { approximately } 9 \mathrm{~cm} \text {. } \\
\text { HOH }\end{array}$ \\
\hline 9 & 6 & A. $\mathrm{R}$. & $\begin{array}{l}\text { Advanced emphysema. } \\
\text { Maximum lung tension } \\
\text { approximately } 8 \mathrm{~cm} \text {. } \\
\text { HOH }\end{array}$ \\
\hline 10 & Many & A. $\mathbf{R}$. & $\begin{array}{l}\text { Advanced emphysema. } \\
\text { Maximum lung tension } \\
<10 \mathrm{~cm} \text {. HOH }\end{array}$ \\
\hline 11 & Autopsy only & A. $\mathbf{R}$. & $\begin{array}{l}\text { Advanced emphysema. } \\
\text { Maximum lung tension } \\
2 \mathrm{~cm} \text {. at autopsy }\end{array}$ \\
\hline
\end{tabular}

* Coefficient of lung tension-change in lung tension, expressed as $\mathrm{cm}$. $\mathrm{HOH}$ pressure $/ 100 \mathrm{cc}$. change in lung volume. In Cases 1-7 lung volume and lung tension changed proportionately.

A. R.-Abnormal relationship between lung tension and lung volume.

as the change in lung tension brought about by $100 \mathrm{cc}$. change in lung size (Table I).

\section{Limitations of the method}

1. The expression of force in terms of $\mathrm{cm}$. $\mathrm{HOH}$ pressure is a relative one. It means grams $/ \mathrm{cm}^{2}$ but the number of $\mathrm{cm}^{2}$ over which the pressure acts is not known. This has important bearing on the appraisal of elastic properties of the lung.

2. The tambours are slow but of similar natural frequency, $3.4 \mathrm{cycles} / \mathrm{sec}$. for the airflow tambour and $9 \mathrm{cy}-$ cles/sec. for the pleural pressure tambour.

3. In each case studied the pneumothorax space was small, nevertheless the pleural pressure on the pneumothorax side may be expected to be higher than that of the opposite side and the greater the degree of collapse, the greater the discrepancy. It was thus found that introducing an additional $500 \mathrm{cc}$. of air slightly increased the 

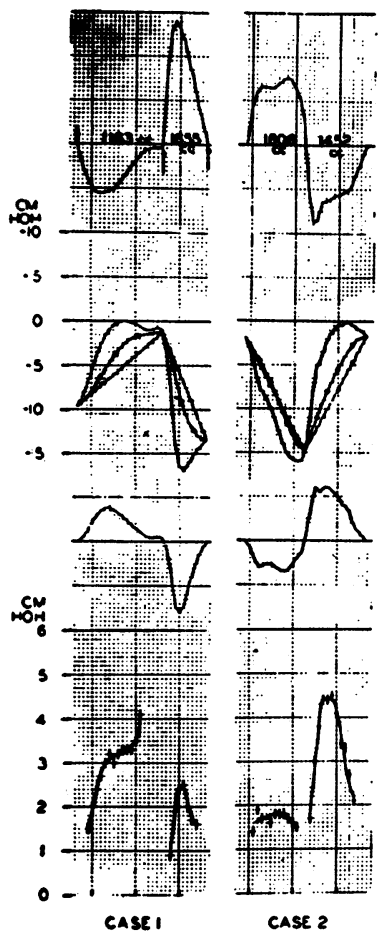

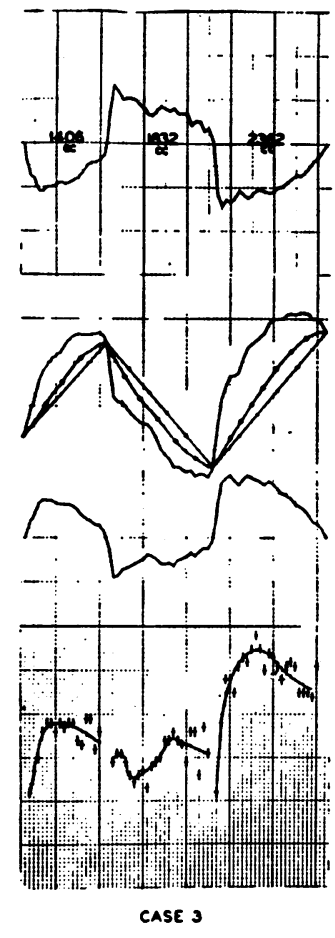

FIG. 4

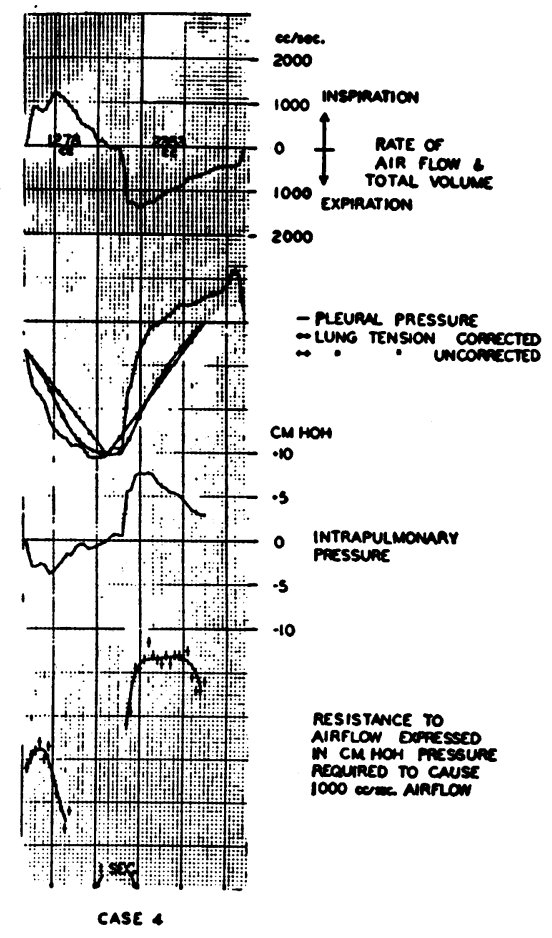

calculated resistance in the airways and slightly decreased the coefficient of lung tension (Table I). The latter is in agreement with the findings of Neergaard and Wirz. The position of the mediastinum is itself a measure of pressure difference between the two pleural spaces and we have observed in fluoroscopy that during expiration the position of the mediastinum remains fixed until the lung on the pneumothorax side deflates to a constant volume, somewhere in the supplemental air range. Further expiratory effort then causes a shift of the mediastinum to the opposite side. With the succeeding inspiration, the mediastinum first moves back to a position close to the midline and then the lung on the pneumothorax side begins to inflate. It appears, then, that difference in pressure between the two sides of the chest is pronounced only in the extreme expiratory position.

4. With small intrapulmonary pressures, accuracy is limited by difficulties in mensuration and by minor fluctuations in both pleural pressure and airflow due to heart beat. This is particularly likely to be the case since small errors in plotting pleural pressure or lung tension can produce large errors in the computation of intrapulmonary pressure (Figure 3 ).

5. Since it was our purpose to study the mechanical factors during hyperpnea it happened that some breaths either began or ended with a positive static pleural pressure. During the portion of the breath when the lungs were exerting an elastic recoil, lung tension could be calculated through knowledge of the coefficient for that patient (Figure 4, Case 4). When the lungs were de- flated, not only to the point where elastic tension was lost, but to the point where they were actually resisting compression, intrapulmonary pressure could not be calculated in this manner. This situation seldom occurred in health but was the rule in advanced emphysema, and is discussed under that section (Case 8).

6. It is obvious that changes in the calibre of the upper respiratory tract could alter the resistance. The larynx of each patient was examined by means of a mirror before a test was made and each patient was instructed to avoid voluntary stridor. Nevertheless, out of a total of more than 90 breaths analyzed in this study, a few were erratic, and variation in the calibre of the upper respiratory tract was thought to be responsible. Indeed, laryngeal closure produces a characteristic aberration of pleural pressure that may be identified from the tracings.

7. Most students of emphysema have commented on the danger of establishing pneumothorax in an emphysematous subject, a view to which we subscribe. We felt obliged, therefore, to study only those with emphysema who came to us with spontaneous pneumothorax.

8. At the very beginning of an inspiration or expiration, resistance seems unusually low, particularly if the breath begins abruptly. We believe this to result from sudden change in the size of the dead space. It should be remembered that a change in size of dead space of trachea and main bronchi is not associated with corresponding change in lung size or lung tension. Furthermore, dead space air enters or leaves the airways with less resistance than parenchymal air. Both factors con- 
tribute toward minimizing the computed pulmonary pressure.

9. The forces which bring about ventilation must not only overcome the resistance to flow of air but also the viscosity or viscance (5) of pulmonary tissue itself. The delicate walls of ventilating units are not likely to offer much viscous resistance to deformation but conceivably this factor might contribute, in health, to the elevated expiratory resistance during rapid flow. It could not contribute appreciably to expiratory resistance in emphysema when tissue contraction has virtually. come to a standstill despite the application of great force in the form of a high positive pleural pressure.

Taking the above limitations into consideration, the results may be said to show the trends in resistance reasonably well.

\section{OBSERVATIONS}

\section{Normally functioning lungs}

Case $1, H . K$., age 26, female nurse, came to us with tuberculous disease confined to the apex of the left lower lobe, under treatment with pneumothorax which produced a very slight collapse of the left lung. There was no dyspnea and no sign or symptom of chronic bronchitis, asthma, or emphysema. The estimated normal vital capacity was $3,000 \mathrm{cc}$. (6), the test vital capacity 2,800 cc. with good cooperation. The vital capacity air was expelled in two seconds.

The inspiratory resistance to airflow fluctuated somewhat (Figure 4), tending to be slightly high at the time when the rate of airflow was beginning to subside. This occurred uniformly with the six inspirations analyzed in this patient. Neergaard and Wirz comment on this phenomenon which occurs during inspiration in some patients and which, so far as we know, is without explanation. The general level of resistance was lower during inspiration than during expiration. During the latter, resistance rose sharply in keeping with the rise of intrapulmonary pressure but tended to remain high as the lungs deflated, even though the effort to exhale diminished. There was a direct proportion between lung size and lung tension (Table I).

Case 2, $M . Z$., age 36, a waiter, presented evidence of tuberculous disease confined to the right upper lobe which was subjected to selective collapse by a small pneumothorax. There was no dyspnea. There were no signs or symptoms of chronic bronchitis, asthma or emphysema. The estimated normal vital capacity was 4,400 cc. Prior to receiving pneumothorax treatment the test vital capacity was 4,150 cc. expelled in five seconds.

During inspiration there was a low steady resistance, or in some breaths a slight decrease in resistance. During expiration there was a sharp rise of resistance during the period of high intrapulmonary pressure (Figure 4). There was a direct proportion between lung size and lung tension.

Case 3, P. Z., age 17, female student, had tuberculous disease restricted to the right apex and was being treated by pneumothorax which gave a small selective collapse at the summit. There was no dyspnea. There were no signs or symptoms of chronic bronchitis, asthma or emphysema. The estimated normal vital capacity was $3,200 \mathrm{cc}$. The vital capacity at the time of the test was $3,200 \mathrm{cc}$. expelled in two seconds. After an additional 500 cc. pneumothorax injection, the vital capacity was 3000 cc.

During inspiration, the resistance remained fairly steady. During a gentle expiration, resistance rose slightly in keeping with the rise in intrapulmonary pressure (Figure 4). During a larger, more forceful expiration, resistance rose to higher levels and as the lungs deflated, tended to remain high even though the intrapulmonary pressure declined. Resistance was slightly higher for both inspiration and expiration after injecting $500 \mathrm{cc}$. additional air into the pleural space. There was a direct proportion between lung size and lung tension (Table I).

Case 4, P.C., age 55, male laborer, with tuberculous disease confined to the summit of the right lung, was being treated by pneumothorax which produced a limited selective collapse of the right apex. The patient was not dyspneic and presented no history or signs of chronic bronchitis, asthma or emphysema. The estimated normal vital capacity was $4,200 \mathrm{cc}$., the test vital capacity 3,150 cc., expelled in five seconds with fair cooperation. After injecting an additional $500 \mathrm{cc}$. of air into the pleural space the test vital capacity fell to 2,800 cc. The patient was judged to have normally functioning lungs within the conditions of the experiment, and to be representative of an older age group.

High pulmonary pressure and low lung tension were associated with high resistance to expiratory airflow. After introducing an additional $500 \mathrm{cc}$. 
of air in the pleural space the trends of resistance were the same but at a slightly higher level for both inspiration and expiration. There was a direct proportion between lung size and lung tension (Table I).

Case 5, T.M. This man, age 20, had never had symptoms or signs of bronchitis, asthma, or emphysema. A small therapeutic pneumothorax was present on the right because of a slight tuberculous lesion in the right apex. Vital capacity was 4,200 cc. expelled in three seconds, normal 4,600 cc. Maximum minute ventilation was $172 \mathrm{~L}$. He breathed within a range of high lung tension. This made it possible for him to exhale at rates reaching as high as $3,400 \mathrm{cc} . / \mathrm{sec}$. without resorting to a positive dynamic pleural pressure. Even at the end of a rapid expiration of 2,300 cc. the static pleural pressure was well on the negative side with the result that the only expiratory obstructive phenomenon was an increase in resistance during periods of high pulmonary pressure (Figure 5, A and $B$, expirations only are shown).

\section{Chronic bronchitis}

Case 6, W. H. This colored male, a laborer, age 53, provides an interesting contrast with Case 5 , since W. H., to outward appearance, had normally functioning lungs, but was a heavy smoker and had experienced morning cough and slight wheezing for years. Physical examination confirmed the presence of generalized expiratory wheezing at the end of forceful expiration.

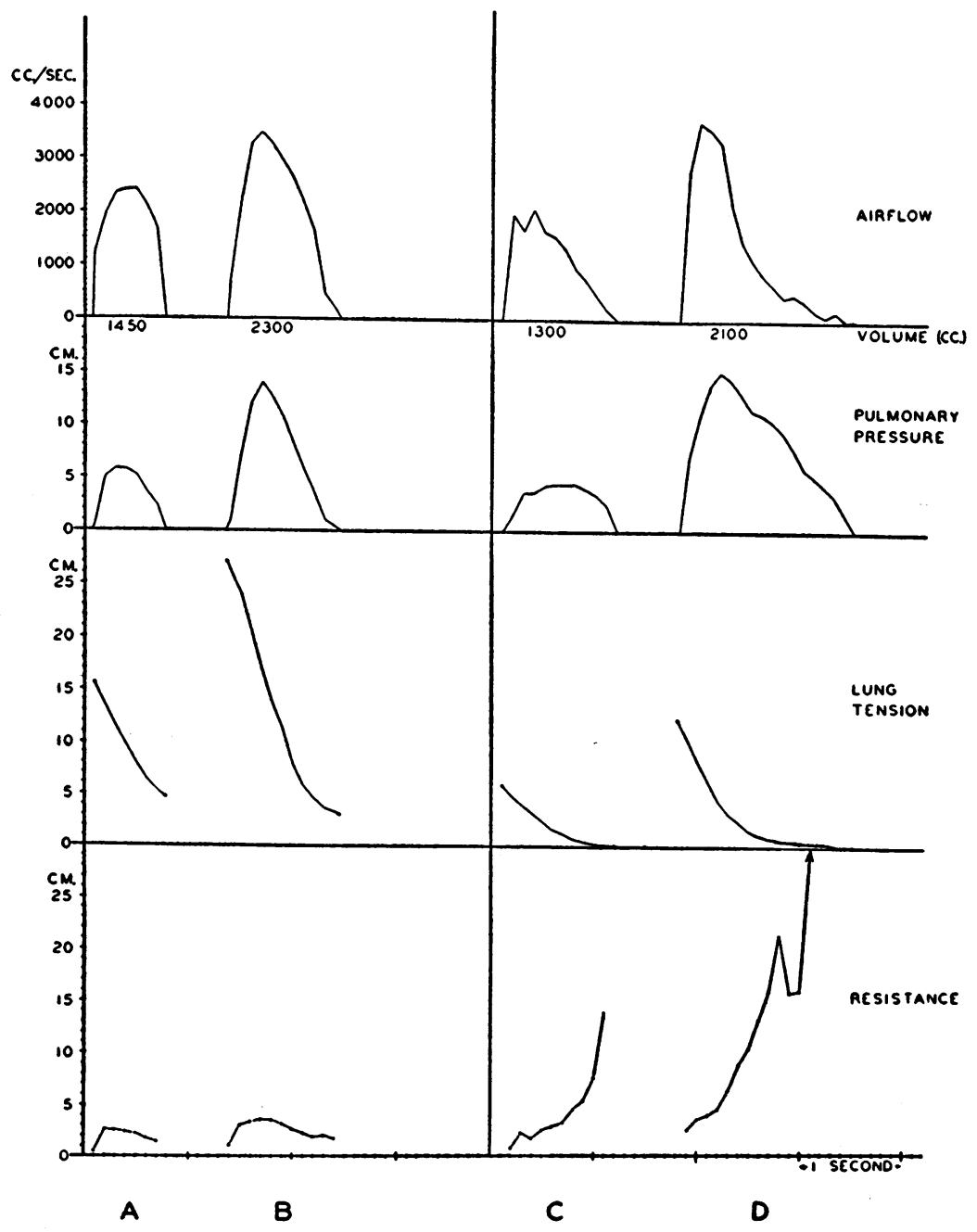

FIG. 5 
There was a slight tuberculous lesion at the right apex and a small selective pneumothorax was begun on that side without causing the patient any subjective respiratory embarrassment. The maximum minute ventilation was $105 \mathrm{~L}$. The vital capacity air was expelled in nine seconds. The elastic tension of the lungs was low (Table I and Figure $5, C$ and $D$ ), so that high rates of expiratory airflow were accomplished by employing a pulmonary pressure in excess of lung tension. In other words a positive pleural pressure was required. There was, however, a direct relation between the lung size and lung tension. The overall resistance to expiration was greater than in Case 5 as may be seen from the areas beneath the pulmonary pressure curves. Early in expiration, while lung tension was still adequate, W. H. did not experience excessive resistance, but at comparable lung tensions, in expirations $\mathrm{C}$ and $\mathrm{D}$, high pulmonary pressure met with proportionately high resistance. As lung tension approached zero, resistance became virtually absolute.

Cases 5 and 6 illustrate the interrelationship between lung tension, pulmonary pressure and narrowing of the airways due to bronchitis. If lung tension is adequate and the airways fully patent, a high pulmonary pressure gives excellent rates of airflow, and narrowing of airways due to gradually decreasing lung tension is slight. If there is partial obstruction of the airways there is greater tendency for high pulmonary pressure to be associated with high resistance. In the latter case, resistance becomes very high if lung tension approaches zero. In both patients resistance to airflow during inspiration was uniform and normally low.

\section{Isolated pulmonary bullae}

Case $7, L$. O., age 44 , garage attendant, had not, so far as he was aware, experienced more shortness of breath than his healthy associates until the time of his last illness. The patient developed a malignant tumor of the left kidney with metastasis to the head of the right seventh rib, and adjacent spine, and came to us with a traumatic pneumothorax on the right, brought about by a diagnostic thoracentesis. Our examinations further disclosed the presence of a giant bulla occupying the entire right upper lobe and another involving the greater portion of the left upper lobe.

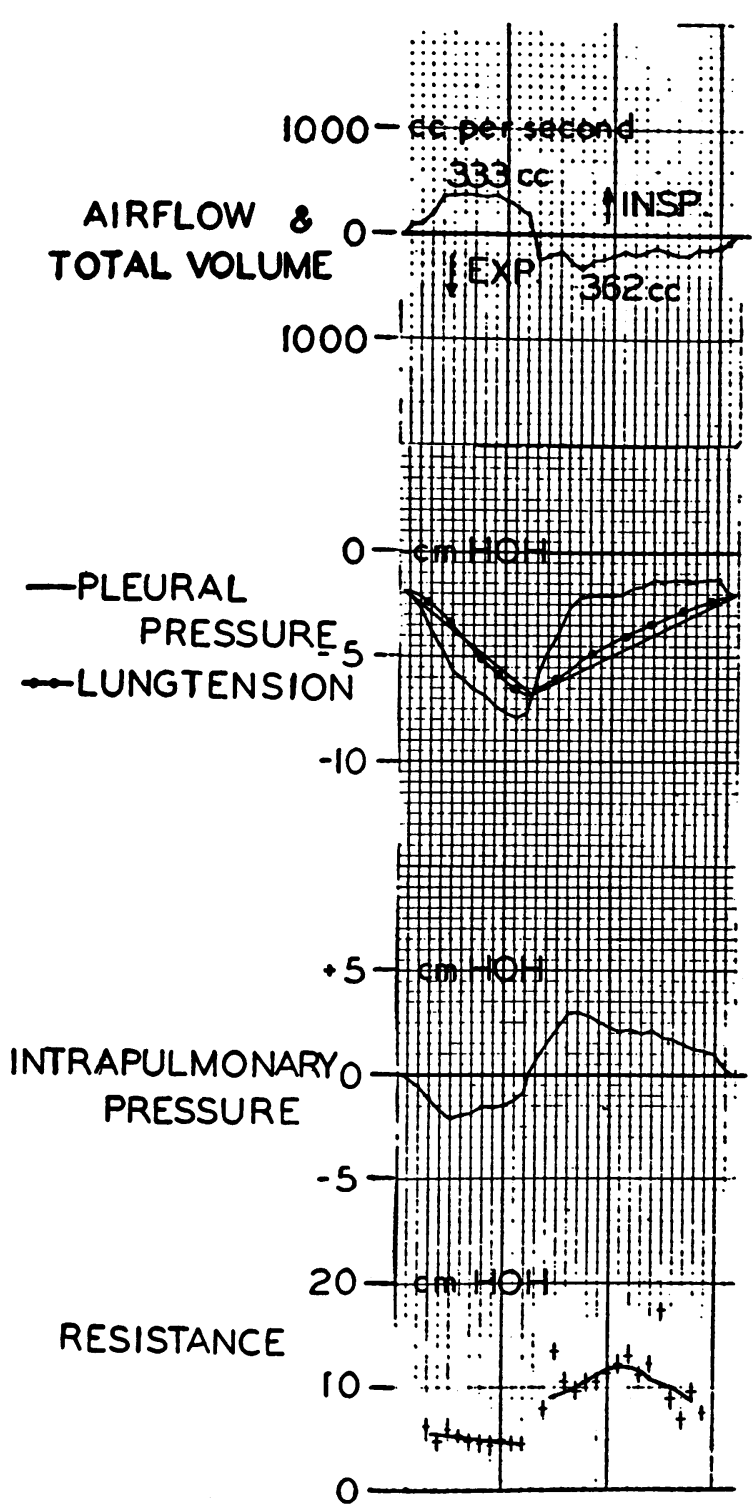

FIg. 6

Breath sounds on the right were obscured by pneumothorax, those on the upper left by the bulla. Over the remainder of the chest the breath sounds were barely audible at rest and became normally intense with forced breathing.

At fluoroscopy the left lower lobe and the partially collapsed right middle and right lower lobes appeared to ventilate well. The bulla in the right upper lobe changed size very little with respiration, but that in the left upper lobe inflated and deflated about in proportion to the rest of the left lung. 
The experiment was performed after the right lung had nearly re-expanded, the needle being inserted in the right fifth interspace. Because of pain the breaths employed were small and not forceful. During inspiration the resistance to airflow was quite uniform but at a higher level than normal (Figure 6). During expiration the resistance rose to around $12 \mathrm{~cm}$. $\mathrm{HOH}$ pressure. The coefficient of lung tension was significantly higher than normal (Table I).

The immediate cause of death was bronchopneumonia. Autopsy confirmed the presence of bulla formation in both upper lobes. That on the left inflated and deflated readily. Deflation of the bulla on the right required firm pressure and was accompanied by a wheezing sound. There were three smaller bullae along the cardiac lapet of the left upper lobe which had been unrecognized during life, one of which could not be deflated even by extremely forceful squeezing. The latter, along with a block of adjacent lung, was excised and the supplying bronchiole cannulated with a small needle. A small needle was also tied into the peripheral wall of the bulla itself. Air flowed readily through the bronchiole into the bulla but could not be made to pass in the opposite direction. The bulla was then filled with hot colored gelatin through the bronchiole and, after cooling, the entire structure was sectioned with a razor. The gelatin had found a complicated passageway through the fragmented lung tissue in the base of the bulla. Since the gelatin was forced in by pressure within the passageway it tended to distend the spaces. It could readily be seen how pressure from without would tend to close the same passageways and prevent the egress of air from the bulla.

The floors of all the bullae in this case were made up of fragmented lung parenchyma, some of them thread-like remnants of blood vessels. Nowhere could a bronchus be traced directly into the bulla space itself; air always had to pass through intervening broken down lung parenchyma. This circumstance has been encountered a number of times in the past both by the late Dr. Leroy Gardner (7) and myself. The result is that air can usually enter a bulla readily but it is a matter of chance whether it can be expelled. Loeb (8) cites the failure to express air from a bulla at $300 \mathrm{~mm}$. $\mathrm{Hg}$ pressure and the finding that the contained gas was almost entirely nitrogen. Breakdown of lung parenchyma creates expiratory check valves while leaving inspiration unimpeded.

The lower lobes of each lung and the right middle lobe were the site of acute bronchopneumonia. Approximately half the bronchioles on section were filled with pus. The entire inflammatory process appeared to be a terminal event. In these portions of the lung there was no morphologic evidence of emphysema either grossly or by microscopic examination.

We concluded that at the time of the experiment the lower portion of the lungs was ventilating normally or nearly so. The bullous areas on the other hand contributed relatively little to the ventilation. Particularly during quiet breathing it was likely that ventilation was restricted to a less than normal amount of lung parenchyma. This probably accounts for the high resistance to airflow and the high figure for coefficient of lung tension.

\section{Advanced generalized obstructive emphysema}

Case 8, G. B., male, age 41, a laborer, had since youth suffered from a severe chronic productive cough, wheezing and dyspnea. The symptoms. were aggravated by frequent protracted attacks of acute bronchitis. For four years he had been unable to work because of dyspnea. The patient was well nourished, somewhat cyanotic, dyspneic on the slightest exertion, but not orthopneic. The chest was fixed in the inspiratory position. There were generalized expiratory rhonchi. The breath sounds were inaudible at rest and scarcely audible with forced breathing. There was no evidence of cardiac or tuberculous disease. The estimated normal vital capacity was $5,000 \mathrm{cc}$. The test vital capacity was $2,100 \mathrm{cc}$. and the patient required ten seconds to expel this amount of air. The maximum minute ventilation was $32 \mathrm{~L} . / \mathrm{min}$. The findings were typical of generalized bronchitis and advanced emphysema.

The patient developed a spontaneous pneumothorax on the left side and after the air had been almost entirely reabsorbed, the experiment was performed. The needle was inserted in the left fourth interspace. The data showed striking deviations from the normal (Figure 7). 


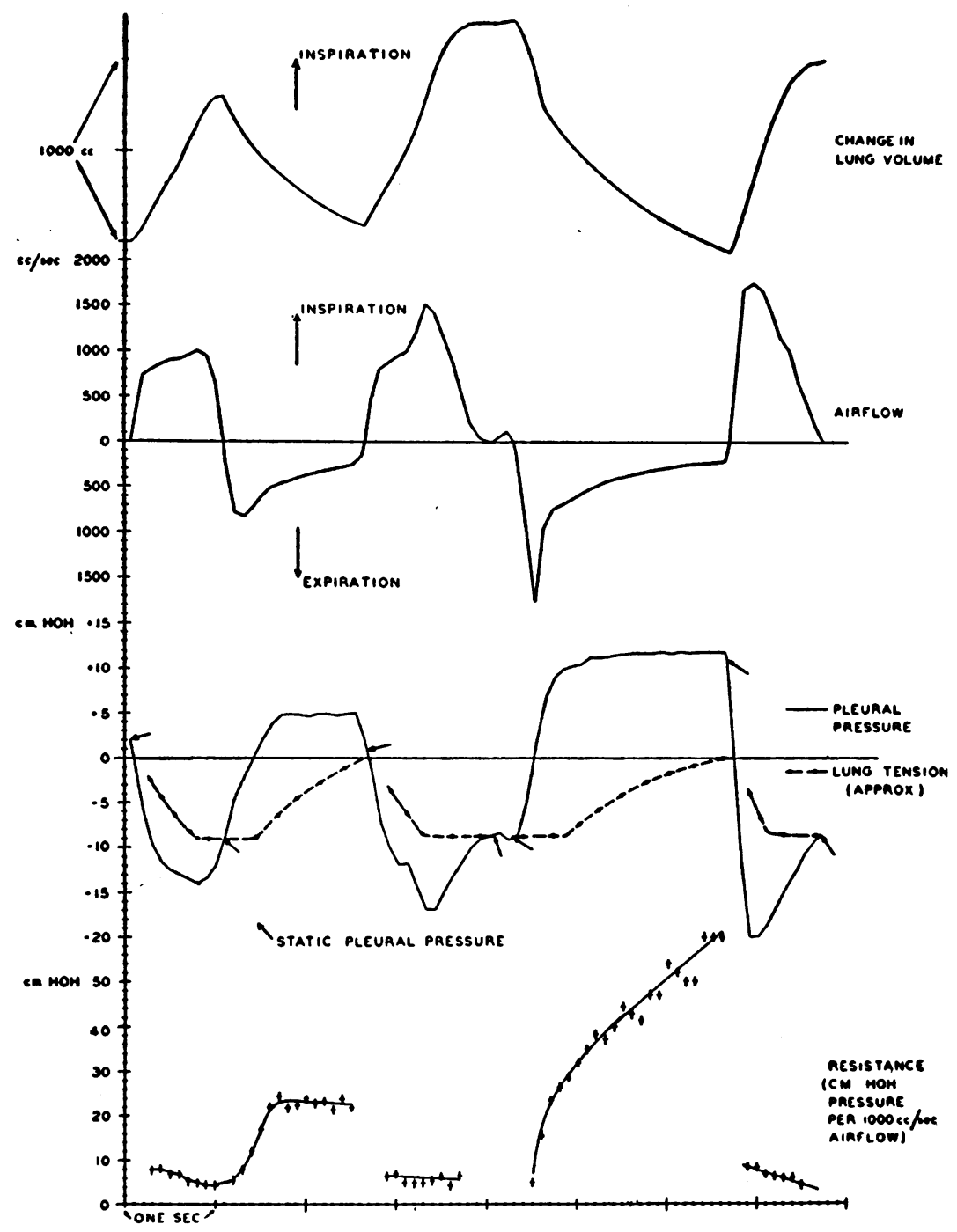

FIG. 7

Reasonably good rates of airflow occurred during inspiration and the rate could be augmented by greater effort though the resistance in the airways was clearly higher than normal. During gentle expiration a fair initial rate of airflow was obtained but the rate decreased despite a sustained positive pleural pressure. If expiration was forceful there was an initial good rate of airflow then a sudden decrease in rate. The decrease of airflow was more conspicuous the greater the effort to expel air. A pleural pressure of $+25 \mathrm{~cm}$. $\mathrm{HOH}$ brought about rates of airflow in the range of only 200 to $300 \mathrm{cc} . / \mathrm{sec}$. As further evidence of the expiratory check valve mechanism in this case it was shown that a sustained positive pleural pressure of 11 to $12 \mathrm{~cm}$. HOH failed to deflate the lungs much farther than a gentle effort (Figure 7).

Accurate measurement of pulmonary pressure was made difficult by the abnormal relationship between lung volume and lung tension (Figure 8). In the first place, expiration usually ended with a positive static pleural pressure. Since emphysema is characterized by a large residual air, and in this patient the lungs were visibly distended even at the end of expiration, we concluded that the positive static pressure was being exerted against a cushion of trapped intrapulmonary air. Release of such positive pleural pressure found lung tension to be zero at this lung size. 


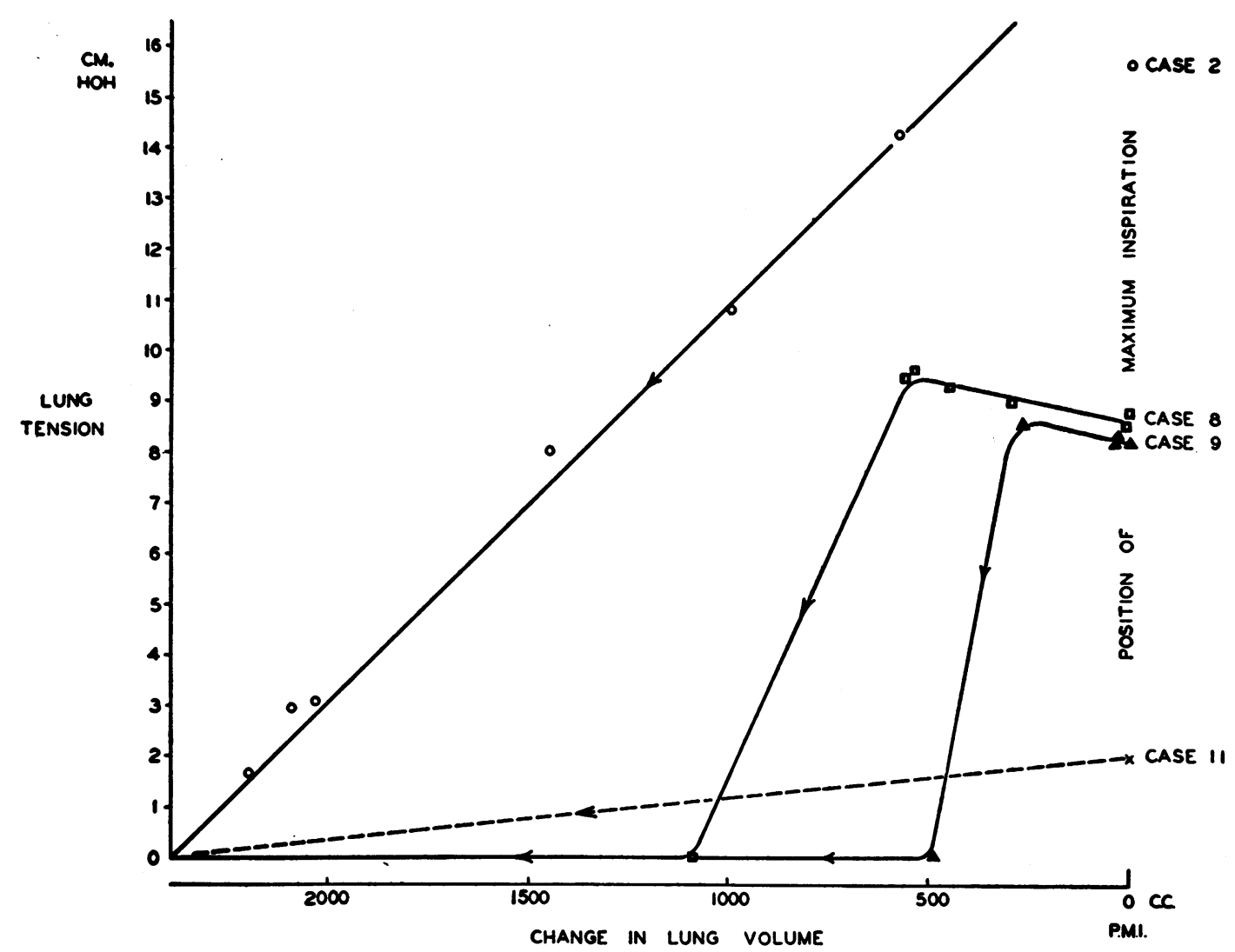

FIg. 8

Inspiration was marked by a sudden rise of lung tension to about $9 \mathrm{~cm}$. $\mathrm{HOH}$ where it remained to the point of maximum inspiration. This relationship between volume and tension is abnormal. In Figure 8 a normal relationship is illustrated for comparison. In this case of advanced emphysema, measuring from position of maximum inspiration, air could be expelled under satisfactory lung tension to the extent of about $800 \mathrm{cc}$. Thereafter lung tension fell quickly to zero. During such a period expiratory airflow suddenly shut off, despite mounting, positive pleural pressure.

Derived from Figure 8, approximate lung tension curves were interpolated in Figure 7, and resistance determined. It is noteworthy that inspiration is relatively unimpeded while obstruction to expiration is extreme following the initial period. The terminal airflow can be regarded as mere leakage. The above findings can only be explained by an expiratory check valve mechanism.

Case $9, H . R$. , male, age 64, laborer, had suffered from a harrassing, productive cough, wheezing and dyspnea for 35 years. He was admitted, critically ill, with a typical, clinical picture of bronchitis and advanced emphysema complicated by spontaneous pneumothorax on the left. $\mathrm{He}$ was treated by means of an intrapleural catheter and water trap. Tests were done two days later when the pleural space was almost obliterated. Pleural pressure was optically measured through the catheter.

The results were very similar to those of Case 8 (Figure 9). Lung tension in the position of maximum inspiration was approximately $8 \mathrm{~cm}$. $\mathrm{HOH}$. Measuring from a position of maximum inspiration the expulsion of approximately $600 \mathrm{cc}$. of air brought about a zero lung tension (Figure 8). At the same time dynamic pleural pressures became highly positive, airflow diminished to mere leakage, and approximate resistance to expiratory airflow rose sharply to ranges of 40 to $60 \mathrm{~cm}$. $\mathrm{HOH}$ per $1,000 \mathrm{cc}$./sec. airflow.

The left lung re-expanded but spontaneous pneumothorax recurred two weeks later and the patient died with left empyema complicated by a 


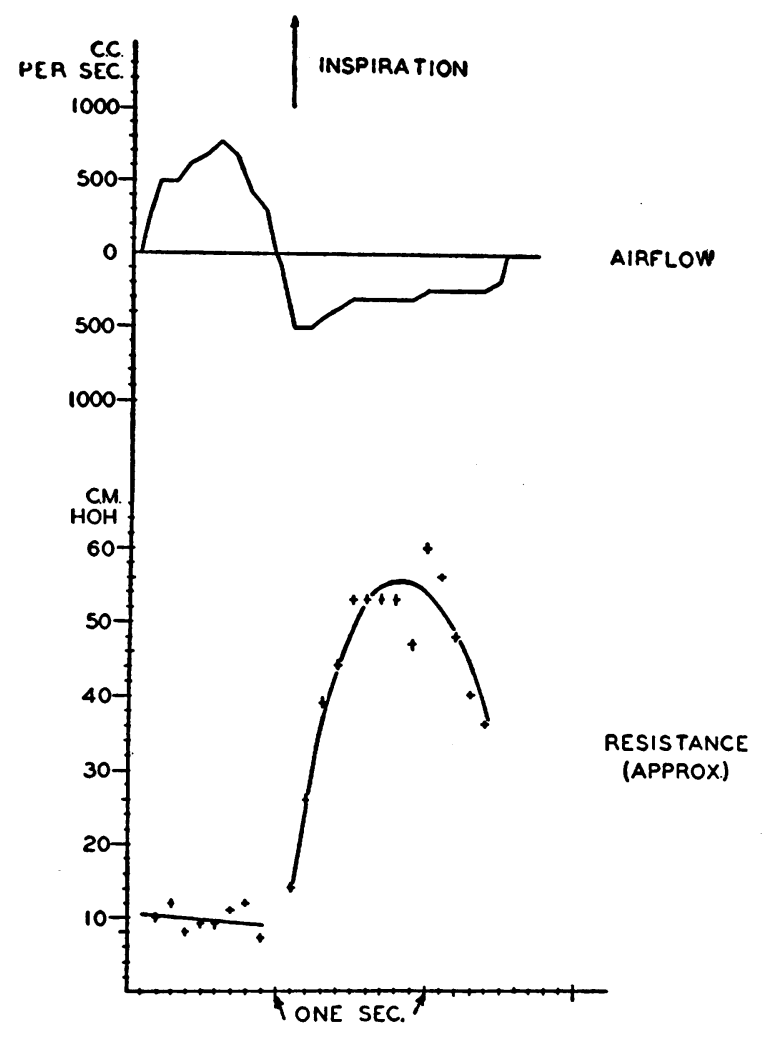

FIG. 9

massive hemorrhage from a bleeding peptic ulcer. On opening the thorax at necropsy the right lung gradually deflated; there were numerous bullae at the apex and along the margins of the upper lobe. The left lung was adherent to the chest wall at the base due to an organizing pleuritis with effusion. A $1 \mathrm{~mm}$. perforation of a small apical bulla accounted for the recurrent pneumothorax over the upper third of the left lung. The left lung was not suited to study of tension because of the pleuritis. The right lung was repeatedly filled with air and allowed to empty, a maneuver which usually promotes even distribution of air in the filling of excised lungs. In this case, however, if one began with a flaccid, but partially inflated state, the instillation of $100 \mathrm{cc}$. units of air brought about erratic pressure response, varying from +4 $\mathrm{cm}$. $\mathrm{HOH}$ to 0 . This is to be expected in the inflation of lungs having impaired tension, since a consistent rise in tension during inflation of the lungs is necessary to insure even distribution of air.

After 1,500 cc. of air had been instilled the pressure became more uniform at +7 to $+9 \mathrm{~cm}$.
$\mathrm{HOH}$. Parenchymal rupture was suspected at $2,000 \mathrm{cc}$. inflation and definite interstitial emphysema was noted near the lung root at $2,400 \mathrm{cc}$. At the point of rupture the pressure was still in the range of +7 to $+9 \mathrm{~cm}$. $\mathrm{HOH}$.

So far as this method of study of excised lungs will permit, the findings resembled those during life. Experience with the excised lungs of man, dog, rabbit, and guinea pig indicates that volume/ tension relationships are much the same in life as in death. The studies of excised human lungs, here reported were carried out within 10 hours after death, at room temperature and without embalming.

It should be noted that in Case 9, (a) the lung did not exert consistent tension till inflation approached the point of rupture, $(b)$ maximum tension was abnormally low, in the range of +7 to $+9 \mathrm{~cm}$. HOH, (c) the lung was abnormally voluminous.

Section of the lungs after imbedding with hot gelatin disclosed moderate parenchymal breakdown, most pronounced in peripheral areas which were the site of bulla formation.

Case 10, J. M., age 43, male, hospital attendant, stated that for many years he had suffered from chest colds with cough, expectoration, wheezing and dyspnea. The patient came to us with moderately advanced bilateral ulcerative tuberculosis. In addition he presented dyspnea in excess of what one would expect from the tuberculous disease alone. The chest was fixed in an inspiratory position. There were generalized expiratory rhonchi. The vesicular breath sounds were inaudible at rest and could be intensified only slightly with rapid inspiration. The estimated normal vital capacity was $4,350 \mathrm{cc}$. The test vital capacity was $2,650 \mathrm{cc}$., and the patient required ten seconds to expel this amount of air. The maximum minute ventilation was $28 \mathrm{~L} . / \mathrm{min}$. A diagnosis of complicating generalized bronchitis and advanced emphysema was made.

The patient developed a spontaneous pneumothorax on the right side, and the author examined the patient within a few minutes after he began to complain of excessive dyspnea. The patient was cyanotic, desperately short of breath and was exerting maximum effort at both inspiration and expiration. Breathing was nevertheless shallow, rate $30 / \mathrm{min}$. The right side of the chest was 
considerably more distended than the left. The mediastinum was deviated only slightly to the left. Breath sounds, rales and rhonchi were inaudible on the right. Oxygen was given and $\mathrm{X}$-rays quickly taken at the bedside. Despite over-distension of the right side of the thorax the lung was uniformly within 1 inch of the chest wall. After inserting a needle barely through the parietal pleura and withdrawing $1,100 \mathrm{cc}$. of air, the lung could be felt touching the needle point. The initial pressure in the pleural space was slightly negative during inspiration $(-2$ to $-10 \mathrm{~cm}$. $\mathrm{HOH}$ ) but during expiration the pressure rose to +25 to $+40 \mathrm{~cm}$. $\mathrm{HOH}$. Since repeated aspirations gave only temporary relief a rubber tube was inserted and the lung expanded by continuous suction.

The patient was too ill to permit an elaborate study of ventilatory mechanics but it was clear that the patient could not effectively expel air from the lungs by employing a highly positive intrapleural pressure, despite the fact that the lungs were over-inflated. The over-distended condition of the thorax, particularly the right side, and the slightly negative intrapleural pressure during inspiration suggest that inspiration was less impeded than expiration and that the inspiratory difficulty arose from the fact that the chest wall and diaphragm had already attained a maximum inspiratory position. It should be noted also that the most negative pleural pressure at any time recorded in this patient was $-10 \mathrm{~cm}$. $\mathrm{HOH}$ indicating a decrease in elastic tension of the lung similar to that in Case 8.

Case $11, A$. B., male, age 46 , a truck driver, stated that for many years he had suffered from an extremely harassing productive cough, accompanied by wheezing in the chest. During the 10 years prior to coming to us dyspnea gradually had become totally incapacitating but during the same period cough and expectoration had slowly subsided.

The patient was thin, cyanotic and dyspneic lying at rest. The chest was fixed in inspiration. Fluoroscopy showed that the dome of the diaphragm was at the level of the 12 th rib posteriorly during inspiration and could not be measurably elevated during the most forceful expiration. The vesicular breath sounds were inaudible both at rest and with forced breathing. There were a few distant scattered sibilant expiratory rhonchi. The abdominal muscles became boardlike in contraction during expiration but were flaccid during inspiration. The outlet of the right ventricle was slightly prominent but there were no signs of myocardial failure. There was no tuberculous disease of the lungs. The findings were typical of bronchitis and extremely advanced emphysema conforming to a type described by Alexander (9) wherein the symptoms of bronchitis or asthma have largely subsided but the patient remains incapacitated by the structural damage in the lung parenchyma itself. Death was due to respiratory failure.

At autopsy the chest was opened with care to prevent perforation of the visceral pleura. During the course of 15 minutes while the lungs lay undisturbed in situ the right lung slowly deflated to about one half its original size and the left lung deflated an estimated $10 \%$. Further collapse occurred during the removal of the organs. A cannula was fastened in the trachea and air instilled by means of a pneumothorax apparatus. The left lung inflated more readily than the right but the arresting feature was the apparent diminution of elastic recoil. After instilling $5,000 \mathrm{cc}$., lung tension was only $2 \mathrm{~cm}$. HOH. Under no circumstance could air be removed from the lungs rapidly, even with a pump. The latter merely caused visible collapse of the airways.

Portions of the lungs were filled with hot gelatin to give rigidity and the lungs were then fixed. Gross and microscopic study confirmed the presence of generalized, extremely advanced emphysema. Air spaces varied in size up to $2 \mathrm{~cm}$. in diameter or larger. Thick, cleared, unstained sections of the gelatin imbedded lung showed that remaining walls were frequently perforated with gaping irregular holes so that spaces were freely intercommunicating. Thread-like structures traversed the spaces. Microscopically they were fibrous cords with minute central vessels, evidently vascular remnants. Elastic tissue stains showed an adequate amount of elastic tissue in such air space walls as remained. Histologically the picture was one of a loss of lung parenchyma rather than a loss of elastic tissue. The destruction of lung parenchyma was so marked as to 
deprive the small bronchi and bronchioles of the surrounding elastic framework which would normally exert a force tending to distend them. An attempt to exhale, creating a positive pressure in the large intercommunicating spaces surrounding such a bronchiole could not fail to collapse and thereby occlude it.

\section{Observations of the trachea during cough}

If a slender person is fluoroscoped in an oblique position the outline of the trachea can be clearly discerned. If the patient is asked to cough energetically, momentary collapse of the thoracic trachea is observed as the glottis opens. Check valve closure is the only conceivable explanation for this phenomenon. It is brought about by the high intrathoracic pressure made possible by the closed glottis. The same phenomenon has been observed by Lell (10) through the bronchoscope in status asthmaticus. In this case generalized obstruction of the smaller airways permits a high, expiratory intrathoracic pressure and the membranous portion of the trachea herniates into the lumen, completely occluding it.

\section{DISCUSSION}

Laennec concluded that the essential lesion in emphysema was obstruction of the smaller airways. To explain why obstruction should lead to abnormal distention of the lungs, he asserted that the muscles of inspiration are more powerful than those of expiration, a view that is commonly held to this day. Nevertheless, greater force can be exerted against a column of mercury by expiratory effort than by inspiratory effort $(11,12)$, a point which we have verified. Moreover, in a tubular system, airflow should bear a more or less direct relationship to pressure, all else being constant; yet in emphysema expiratory airflow is not proportionately improved by greater effort. Greater force is met by greater resistance. Force, therefore, would be adequate were it not for the singular character of expiratory resistance to airflow.

Christie $(2,3)$ and others concluded that the essential disturbance in emphysema is a loss of pulmonary elasticity. Loss of elasticity could bring about expiratory impedance by a mechanism described below, but it is not the sole mechanism. The syndrome of relatively free inspiration, impeded expiration and increased residual air occurs in at least two conditions when elasticity is intact; during an acute paroxysm of asthma (13) and in lung distal to a local bronchial obstruction such as that resulting from tuberculous bronchitis. With release of the obstruction, the syndrome disappears. We, therefore, must seek explanation for ventilatory disturbance in emphysema in the extrinsic mechanical factors as they affect the calibre of the airways rather than inadequate expiratory force or loss of pulmonary elasticity per se.

Extrinsic force can have a decided effect on calibre of the airways. Momentary narrowing of the trachea as the glottis opens in the expulsive phase of a forceful cough, and occlusion of the trachea by herniation of the membranous portion during expiration in severe asthma are the evident results of a pronounced pressure gradient between the inside and outside of the airway. An unmistakable check valve mechanism is in effect.

Neergaard and Wirz attributed greater expiratory resistance to this mechanism, reasoning that the pressure in the parenchyma surrounding an airway would be greater than that within its lumen, thereby causing it to collapse (4).' Our findings are in keeping with this theory but indicate that the operation of an expiratory check valve mechanism is subject to the interrelated factors of lung tension, pulmonary pressure, rate of airflow and intrinsic disease of the airways, in such a way as to minimize the obstruction in health and render it virtually absolute in certain forms of emphysema.

Consider first a hypothetical segment of normal lung at a moment of apnea with a pleural pressure of $-15 \mathrm{~cm}$. HOH. All structures from the pleura to the hilum are part of a continuous elastic system; all, including the walls of the airways, are subject to the distending force of $15 \mathrm{~cm}$. $\mathrm{HOH}$; all are exerting an equivalent counter tension. Canalicular pressure is atmospheric.

Expiration is initiated by a rise of pleural pressure and an equivalent rise of pulmonary pressure. However, there is a descending canalicular pressure along the path of airflow and the force distending the airway becomes $15 \mathrm{~cm}$. HOH 
minus the difference between parenchymal and canalicular pressure at a given point in the airway. Elastic equilibrium can be re-established by narrowing of the airway and relaxation of its walls to the point of equal counter tension. The higher the pulmonary pressure the steeper the pressure gradient and the more the narrowing. Narrowing of the airway itself exaggerates the pressure gradient. Potentially a vicious circle is present.

Thus far we refer to passive narrowing of airways similar to diastolic arterial narrowing. Actual collapse at a given point in an airway could occur only if pulmonary pressure exceeded lung tension plus canalicular pressure at that point. Since pulmonary pressure is equal to the difference between lung tension and pleural pressure, the latter would have to be positive. Active expiratory effort, such as that occurring in coughing or sneezing, would be required. Passive expiration would be ineffective.

As the lung deflates lung tension decreases. This, itself, leads to passive narrowing of the airways. As lung tension approaches zero, pulmonary pressure can be maintained only by resorting to positive pleural pressure. Thus, decline of forces tending to maintain patency of the airways inevitably augments the forces tending to actively narrow them. The stage is set for expiratory check valve closure when expiration is carried to the point of zero lung tension.

Despite limitations imposed by the indirect method of measuring pulmonary pressure and lung tension the experimental findings in normal expiration agree with those postulated in the hypothetical segment. The general level of resistance to airflow in a given case is higher in expiration than inspiration, and is augmented by high pulmonary pressure. As lung tension diminishes the resistance tends to remain high despite declining pulmonary pressures. These changes, though slight, we regard as important indications of a potential check valve mechanism even in health.

Serious expiratory obstruction in health is averted, probably by the following mechanisms :

1. It is doubtful that pulmonary pressure is uniform from pleura to hilum. If it were, the air spaces near the hilum would deflate sooner than those at the periphery, thereby establishing a parenchymal gradient parallel to that in the airway. Such local deflation would, however, cause local decrease in lung tension.

2. The airways subjected to greatest stress in forceful expiration are armored against collapse by cartilage.

3. Resistance to airflow in the normal thoracic airways is slight and the pressure gradient thereby at a minimum. By measuring pharyngeal pressure it can be determined that most of the resistance to airflow in health is in the upper respiratory tract.

4. The healthy patient ventilates the lungs within a range of lung tension which permits adequate expiratory airflow without resort to positive pleural pressure. Thus Case 5 attained rates of $3,500 \mathrm{cc} . / \mathrm{sec}$. by passive recoil alone, surely enough for physiological needs, even in exercise. By positive expiratory effort a healthy man can attain rates of $10,000 \mathrm{cc} . / \mathrm{sec}$. or better, a remarkable achievement considering the complex structure of the lung, and one which is made possible only by the delicate balance of forces which maintain the calibre of the airways, of which the most important is lung tension.

There are two common disease conditions which can disturb this balance of forces: (1) intrinsic obstruction of the airways, (2) loss of lung tension.

1. Intrinsic obstruction of the airways: Regardless of the nature of the lesion, whether it be tumor, local inflammation, generalized bronchitis, or a paroxysm of asthma, an intrinsic lesion partially obstructing the airways produces two effects.

a. Fall of pressure along the course of the obstructed airway is more pronounced than normal.

b. Greater than normal pulmonary pressure is required to produce a given rate of airflow.

In turn, both circumstances predispose to check valve narrowing of the airway. It is understandable, therefore, that if the degree of resistance is sufficient, expiratory impedance, premature arrest of expiration and increased residual air are 
common to all the above disease conditions. It is likewise apparent why a shift of the mediastinum in unilateral obstructive emphysema, due to a local bronchial lesion, is best elicited by a forceful expiratory effort. Closure of the bronchus at the site of such a lesion, early in expiration is described by Jackson (14). If bronchioles are the principal site of the obstruction, a similar closure would be anticipated in them.

In obstructive pulmonary disease two compensatory mechanisms may be employed: $(a)$ The avoidance of rapid expiratory airflow, and $(b)$ breathing so far as possible, within a range of high lung tension $(13,15)$. Both are employed during a paroxysm of asthma.

2. Loss of lung tension: In two forms of emphysema intrinsic obstruction of the airways may be slight or even absent, despite extreme pulmonary insufficiency with typical expiratory impedance and premature arrest. One form is a sequel to allergic asthma wherein the bronchial factor after many years has ceased but the patient suffers from sustained crippling emphysema which does not respond to adrenalin and is characterized at necropsy by advanced breakdown of the parenchyma. Case 11 is typical.

In such an instance sections along the bronchioles leave no doubt about the nature of the expiratory obstruction. The bronchioles are patent but their delicate structure, the tenuous remnants of parenchyma separated by gaping holes about their circumference and the evident loss of lung tension on actual measurement, presents a mechanical situation which can be likened to that of a leaky rubber valve from a basal metabolism apparatus. Such a valve-like bronchiole would conduct expiratory air under low external pressure but an attempt to increase airflow would inevitably lead to narrowing. Such bronchioles, unprotected by cartilage and unsupported by lung tension would be expected to close completely under pressure once any degree of narrowing occurs. With low or absent lung tension, shut-off of airflow typically becomes more sudden the greater the applied force (Cases 6, 8, 9). Furthermore the shut-off occurs under pressures which would be insufficient to materially collapse the major airways, again suggesting that the bronchiole is the site of the closure.
Intrinsic obstruction would, of course, increase the tendency of the unsupported bronchiole to collapse, but collapse would occur even in the absence of intrinsic obstruction provided sufficient pulmonary pressure is applied.

The second form of emphysema in which the unsupported bronchiole plays a part, regardless of intrinsic obstruction, is that complicating fibrosis of the lung, exemplified in conglomerate silicosis. Emphysema in these cases may be most severe, and without history of antecedent bronchial disease. Again, necropsy characteristically shows advanced breakdown of the parenchyma which must result in decreased lung tension and loss of bronchiolar support.

\section{Pulmonary elasticity}

Christie demonstrated the direct relationship between lung volume and lung tension in health, but in two cases of advanced emphysema lung tension increased proportionately at first, then as inflation continued, lung tension tended to level off. In the present study lung tension in four cases of advanced emphysema leveled off between 2 and $10 \mathrm{~cm}$. HOH pressure. There can be no doubt that lung tension is decreased in these cases but we are not justified in saying that the remaining tissue in such lungs had necessarily lost elasticity. In the distension of hollow structures pressure measured in $\mathrm{cm}$. of $\mathrm{HOH}$ does not directly express the stress nor does volume measure the strain. For example, the expression $\mathrm{cm}$. $\mathrm{HOH}$ pressure means gm. per $\mathrm{cm}^{2}$ The area over which this pressure is exerted, and therefore the total force, is unknown, but we do know that the area increases as the structure distends. In the distension of hollow spheres made of thin, good quality rubber, pressure rises to a peak (the "hump") and then levels off or slowly declines as inflation continues (16), a striking parallel to the curve in emphysema.

Since the essential features of emphysema may be present in lungs of normal tension, and loss of tension has been demonstrated only in advanced emphysema where breakdown of parenchyma is either demonstrated or would be expected, and since the tension/volume relationship in advanced emphysema does not necessarily indicate loss of elasticity, we are justified in concluding that the de- 
creased tension is due to breakdown of the parenchyma, a disturbance of architecture, rather than a disturbance of elasticity. To the present there is no valid evidence that the remaining parenchyma of the emphysematous lung is inelastic.

\section{CONCLUSIONS}

1. The method of Neergaard and Wirz can be adapted to show the trends in pleural pressure, lung tension, airflow, pulmonary pressure, and resistance to airflow. Certain errors in the method are noted.

2. During expiration intrathoracic airways behave as check valves. A slight tendency in this direction is detectable even in health, serious obstruction being averted by a number of mechanical circumstances, the chief of which is adequate lung tension.

3. In advanced emphysema check valve obstruction becomes virtually absolute as expiration progresses to the point of zero lung tension.

4. Lung tension in advanced emphysema, measured in terms of $\mathrm{cm} . \mathrm{HOH}$, is reduced and does not bear a direct proportion to lung volume. This is probably the result of breakdown of the parenchyma and does not necessarily imply that the remaining parenchyma is inelastic. Such breakdown of parenchyma leaves the bronchioles unsupported and thereby vulnerable to expiratory check valve closure.

5. The tendency toward expiratory check valve closure is increased by high pulmonary pressure, intrinsic obstruction of intrathoracic airways, and low lung tension.

6. Application of the above principles can explain many of the ventilatory disturbances in obstructive pulmonary disease.

\section{REFERENCES}

1. Cournand, A., Richards, D. W., Jr., and Darling, R. C., Graphic tracings of respiration in study of pulmonary disease. Am. Rev. Tuberc., 1939, 40, 487.

2. Christie, R. V., and McIntosh, C. A., The measurement of the intrapleural pressure in man and its significance. J. Clin. Invest., 1934, 13, 279.

3. Christie, R. V., The elastic properties of the emphysematous lung and their clinical significance. $J$. Clin. Invest., 1934, 13, 295.

4. Neergaard, K., and Wirz, K., Die Messung der Strömungswiderstände in den Atemwegen des Menschen insbesondere bei Asthma und Emphysem. Ztschr. f. klin. Med., 1927, 105, 51.

5. Bayliss, L. E., and Robertson, G. W., Visco-elastic properties of the lungs. Quart. J .Exper. Physiol., 1939, 29, 27.

6. West, H. F., Clinical studies on respiration; comparison of various standards for normal vital capacity of lungs. Arch. Int. Med., 1920, 25, 306.

7. Gardner, L., Saranac Laboratory, personal communication.

8. Loeb, L. M., The etiology of emphysema. Arch. Int. Med., 1930, 45, 464.

9. Alexander, H. L., Emphysema. Proc. Staff Meet., Mayo Clin., 1935, 10, 377.

10. Lell, W. A., Bronchoscopy as an aid in the diagnosis and treatment of allergic pulmonary disease. Arch. Otolaryng., 1946, 43, 49.

11. McLeod, J. J. R., Physiology in Modern Medicine, edited by Bard, P. C. V. Mosby Co., St. Louis, 1938, 8th Ed., 476.

12. Rahn, H., Otis, A. B., Chadwick, L. E., and Fenn, W. O., The pressure volume diagram of the thorax and lung. Am. J. Physiol., 1946, 146, 161.

13. Baldwin, E., Bronchial asthma. Am. J. Med., 1946, 1, 193.

14. Jackson, C. L., Bronchial obstruction. Diseases of the Chest, 1950, 17, 125.

15. Prinzmetal, M., Relation of inspiratory distension of the lungs to emphysema. J. Allergy, 1934, 5, 493.

16. Treloar, L. R. G., Stress strain data for vulcanized rubber. Rubber Chem. and Tech., 1945, 17, 813. 